Esta revista forma parte del acervo de la Biblioteca Jurídica Virtual del Instituto de Investigaciones Jurídicas de la UNAM

\title{
De la calidad de las elecciones a la calidad de las democracias en los estados mexicanos, \\ 2001-2012
}

\section{Nicolás Loza* Irma Méndez**}

\section{Sumario:}

I. La encuesta a expertos en política estatal

II. Elecciones de gobernador: irregularidades y compra y coacción de voto

III. Fuentes consultadas

* Doctor en Ciencias Sociales por el Colegio de México; investigador y profesor de la Flacso México. Toda comunicación dirigirla a loza@flacso.edu.mx.

** Doctora en Gobierno por la Universidad de Essex; investigadora y profesora de la Flacso México. Toda comunicación dirigirla a imende@flacso.edu.mx. 
Esta revista forma parte del acervo de la Biblioteca Jurídica Virtual del Instituto de Investigaciones Jurídicas de la UNAM www.juridicas.unam.mx

\section{Resumen:}

Se expone el estudio a expertos en política estatal realizado en el segundo semestre de 2012 sobre la calidad de los sistemas políticos subnacionales en México, así como describir la incidencia de seis irregularidades electorales, especialmente la compra de voto y la coacción a electores en las elecciones de gobernador ocurridas entre 2001 y 2012 en los estados de la República mexicana. La finalidad fue concentrar y sistematizar parte del conocimiento sobre la calidad y funcionamiento de los sistemas políticos de los estados de la República mexicana en la primera década del siglo XXI; ${ }^{1}$ la descripción de irregularidades es un ejemplo de los usos posibles de la investigación.

El artículo se divide en dos partes, en la primera se hace una descripción del estudio, para que el lector conozca los temas que abarcó y los procedimientos seguidos para generar la información. En la segunda, se ejemplifica de manera descriptiva una parte de la investigación, en este caso, la evaluación de la presencia de irregularidades en las elecciones de gobernador ocurridas entre 2001 y 2012. El trabajo constata que ambos fenómenos asimilables en el concepto más general y analítico del clientelismo, son las dos irregularidades más extendidas en las elecciones locales en México, por lo que limitarlas y volverlas poco relevantes representa un gran desafío para el legislador y los actores políticos involucrados.

Palabras clave: encuesta, política estatal, compra y coacción del voto.

1 El estudio fue posible gracias al financiamiento del Programa de las Naciones Unidas para el Desarrollo (PNUD) y de la Red de Estudios Sobre la Calidad de la Democracia en México de Consejo Nacional de Ciencia y Tecnología (CONACYT), así como de la propia Sede México de la Facultad Latinoamericana de Ciencias Sociales (Flacso). 


\section{La encuesta a expertos en política estatal}

Aunque el estudio de los sistemas políticos subnacionales mediante la encuesta a expertos cuenta con antecedentes en países federales como Rusia, ${ }^{2}$ Kirgistán ${ }^{3}$ y Argentina, ${ }^{4}$ en México no los hay, al tiempo que al menos desde hace dos décadas, la descentralización y democratización brindaron un piso institucional uniforme del que han emergido disparidades entre estos sistemas, por lo que ni la suposición de una democratización subnacional pareja, atada al proceso federal de democratización, ni de 32 islas semiautoritarias en que los gobernadores son virreyes que ejercen su poder de manera ilimitada e idiosincrática, son plausibles. Trabajos recientes sobre elecciones y sistemas políticos subnacionales en México ${ }^{5}$ documentan las diferencias entre estados de la federación, pero normalmente encuentran un límite en el acceso a fuentes de información comparables y exhaustivas, en tanto que de acudirse únicamente a leyes y reglamentos resulta difícil e incluso imposible, incorporar el funcionamiento real de los actores políticos y el uso que hacen de las reglas y de los recursos institucionales. Contar con datos comparables por la coherencia técnica con que se generan, es indispensable para eliminar estos vacíos; la evaluación de expertos es una buena manera de contribuir a subsanar la ausencia.

Nuestra investigación, entonces, partió de este diagnóstico, por lo que nos propusimos entrevistar con un instrumento semiestandarizado a cuando menos siete expertos en política local de cada una de las 32 entidades federativas. El cuestionario constó de 82 preguntas sustantivas, muchas de ellas con respuestas múltiples, que nos per-

2 McMann, Kelly y Nikolai, Petrov, “A Survey of Democracy in Russia’s Regions”, PostSoviet Geography and Economics, 41(3), 2000.

3 McMann, Kelly, Economic Autonomy and Democracy. Hybrid Regimes in Russia and Kyrgyzstan, Nueva York, Cambridge University Press, 2006.

${ }^{4}$ Gervasoni, Carlos, "Democracia, Autoritarismo e Hibridez en las Provincias Argentinas: La Medición y Causas de los Regímenes Subnacionales", Journal of Democracy en Español, vol. 3, 2011.

5 Véase Méndez, Irma y Loza, Nicolás (coords.), La calidad de las elecciones sub nacionales en México, México, Flacso-PNUD, 2013 (en prensa); Martí I Puig, Salvador et al. (eds.), La democracia en México. Un análisis a 10 años de alternancia, Barcelona, Bellaterra, 2011, p. 265. 
Esta revista forma parte del acervo de la Biblioteca Jurídica Virtual del Instituto de Investigaciones Jurídicas de la UNAM

miten conocer las propiedades de las democracias subnacionales en dos fases de su funcionamiento: la de acceso y la de ejercicio del poder. En la dimensión de acceso, se consideraron las subdimensiones de inclusión al sistema político, que involucra la extensión efectiva de los derechos a votar y a competir, de competencia propiamente, que incluye la imparcialidad de la elección, de libertad de expresión y para formar o integrarse a una organización, y finalmente, de efectividad, que implica la influencia sobre las instituciones gubernamentales de la entidad, de los poderes locales no elegidos y de los federales elegidos. En la dimensión de ejercicio se evaluaron los controles institucionales del legislativo, el judicial, las agencias de responsabilidad horizontal y al partido gobernante, en tanto que respecto a los derechos liberales en las entidades, se exploró el estado de los derechos a la libre expresión, al acceso a fuentes de información alternativas, a la información gubernamental, así como de las libertades individuales que incluyen garantías de seguridad, privacidad, para estilos de vida alternativos y para la libertad académica, en tanto que en lo relativo al diagnóstico de la independencia de la sociedad civil, se incluyeron consideraciones sobre la autonomía sindical y empresarial, de las iglesias y de las organizaciones no gubernamentales.

Considerando toda la República, el periodo bajo estudio fue de 2001 a 2012, limitado a no más de seis años en cada estado, con sincronía parcial con un mandato presidencial y entre ellos. El criterio para determinar el lapso de análisis en cada entidad fue que se tratara de la gestión de gobernador más reciente y que estuviera concluida, o que si aún no terminaba, se hubieran realizado las elecciones del gobernador siguiente. Además, como nos interesaba examinar el vínculo estadofederación y gobernador-presidente, acotamos el periodo bajo análisis al que resultara concurrente con la gestión del presidente Calderón, y en este sentido, Baja California fue el único estado cuyo lapso de observación comprendió los años 2001 a 2007; en el ciclo 2002-2008, entraron Campeche, Nuevo León, Querétaro y San Luis Potosí; en tanto que los sistemas políticos de Aguascalientes, Chihuahua, Durango, Oaxaca, Veracruz y Zacatecas fueron evaluados en el periodo 2004-2010; de 2005 a 2011 se reunió información para Coahuila, Guerrero, Hidalgo, Estado de México, Nayarit, Puebla, Quintana Roo, Sinaloa y Tlaxcala, mientras que para el periodo 2006-2012 se consideró a Chiapas, Guanajuato, Morelos, Tabasco, Yucatán y el Distrito Federal, y solamente Jalisco comprendió de 2007 a 2013. Finalmente, peculiaridades esta- 
Esta revista forma parte del acervo de la Biblioteca Jurídica Virtual del Instituto de Investigaciones Jurídicas de la UNAM

tales, la mayoría de ellas asociadas a la sincronización de calendarios electorales introducida en la reforma electoral federal de 2007 y la aplicación de nuestros criterios, hicieron que para Michoacán evaluáramos un periodo de gobierno de cuatro años, de 2008 a 2012, en que sólo hubo una legislatura, en Colima también trabajamos con un periodo de cuatro años, de 2005 a 2009, en Tamaulipas en uno de cinco, de 2005 a 2010 y en Yucatán también con uno de cinco años, de 2007 a 2012.

Nuestro estudio siguió el diseño que Carlos Gervasoni ${ }^{6}$ utilizó para las provincias argentinas, lo mismo con el instrumento de recolección que con la selección de entrevistados, se nacionalizó el fraseo de las preguntas e incorporaron los intereses de investigación de los coordinadores del proyecto así como de los investigadores asociados al mismo, se produjo una versión ligeramente diferente de entrevista que incluyó un módulo sobre la violencia y la acción del crimen organizado en las entidades, amplió las preguntas sobre el poder de los gobernadores y su éxito presupuestal, sobre el equilibrio entre poderes, sobre la identidad ideológica de partidos, gobernantes y entrevistados, y finalmente, en relación con la calidad de los procesos electorales locales. ${ }^{7}$

En los cuestionarios, al evaluar las elecciones, el ejercicio de los derechos liberales o cualquiera otra de las dimensiones referidas, se preguntó sobre procesos recientes pero pasados, en los que no se privilegian las opiniones de los expertos, sino que se solicitan respuestas de hecho. Por ejemplo, en qué medida fue modificada la propuesta presupuestal del gobernador, cuántos partidos dominan el órgano de administración electoral o qué tan probable les parecía que un funcionario de primer nivel fuera investigado y en su caso sancionado, ante denuncias creíbles de corrupción. En la siguiente sección ilustramos

6 Véase Gervasoni, Carlos, “Democracia, Autoritarismo..., cit.

7 En la primera fase del trabajo, Carmina Gutiérrez, Fanny Sleman, Iván Arrazola y Luis Fernando Sánchez, estudiantes del doctorado en ciencias sociales de la Flacso Sede México e integrantes del Seminario de investigación "Democracias y poderes sub nacionales en América latina” conocieron el cuestionario de Gervasoni y propusieron adecuaciones y preguntas de su interés. Posteriormente, circulamos una nueva versión de cuestionario entre colegas interesados en el campo de investigación. Agradecemos las observaciones y sugerencias de Carlos Gervasoni, Diego Reynoso, Juan Olmeda, Álvaro López Lara, Raúl Rocha y César Valderrama, entre otros. 
Esta revista forma parte del acervo de la Biblioteca Jurídica Virtual del Instituto de Investigaciones Jurídicas de la UNAM

fraseo y uso de este tipo de reactivos, con el caso específico de las irregularidades electorales, en particular la compra y coacción de voto.

El procedimiento de campo que seguimos fue el siguiente; en primer lugar, integramos un equipo con maestros, candidatos a doctor e incluso doctores que tienen intereses de investigación en política subnacional en México. ${ }^{8}$ Para cada entidad se elaboró una lista inicial de posibles entrevistados, mismos que, de preferencia, debían ser investigadores con publicaciones en la política del estado en cuestión, integrantes del Sistema Nacional de Investigadores, adscritos a alguna institución de docencia y/o investigación y con grado de doctor en Ciencias Políticas o equivalente. Cuando este perfil fue escaso en la entidad, flexibilizamos uno o más de estos criterios, buscando periodistas, analistas políticos, ex funcionarios, profesores universitarios e integrantes de organizaciones no gubernamentales. La primera lista de cada estado fue puesta a la consideración de al menos un integrante de la Red de Estudios Sobre la Calidad de la Democracia en México u otro académico o experto en la entidad, de manera tal que esa lista fue depurada y/o ampliada. Finalmente, a los primeros entrevistados, les pedimos recomendarnos otros expertos locales, siguiendo un procedimiento de bola de nieve. Aunque para cada caso procuramos que al menos cuatro entrevistas fueran aplicadas de forma personal en la misma entidad, el correo electrónico y las teleconferencias también fueron utilizadas. En varios estados concretamos más de siete entrevistas. ${ }^{9}$

Aplicadas las entrevistas en los estados, cada entrevistador redactó un informe producto de su experiencia y observaciones de campo, mismos que junto con la base de datos y cualquier otro proceso y/o producto asociado al proyecto estarán a disposición de quien lo solicite a partir de marzo de 2013.

8 La maestra Magdalena Aguilar, estudiante del doctorado en ciencias sociales de la UAM-I estuvo a cargo de integrar un equipo de campo, en su mayoría maestros y estudiantes de doctorado en la FCPyS de la UNAM o en la UAM-I, interesados en política local. Agradecemos el trabajo en campo tanto de la maestra Aguilar como de Carlos Díaz González, Gabriela Magdaleno, Melina Castro, Nallely Rubio Jardon, Abel Muñoz, Francisco Javier Navarro, Geovanni Pérez Landa, Javier Rosiles, Joshep Arana, Juan Manuel Rodríguez, Luis Alberto Fernández, Omar Carrillo, Pablo Ranchero y Raúl Figueroa.

9 Al momento de la redacción de este artículo, se contaba con 222 cuestionarios aplicados, capturados y validados, aunque durante enero de 2013 todavía recibiremos algunas entrevistas. 


\section{Elecciones de gobernador: irregularidades y compra y coacción de voto}

En más de 54 preguntas, el cuestionario exploró, en distinta medida, la calidad de las elecciones a lo largo del periodo evaluado, incluso sobre la misma elección en que ganó el ejecutivo local del periodo bajo observación. Se valoraron procesos concurrentes, dos de gobernador, diputados locales y ayuntamientos, y uno más, el intermedio, sólo de diputados locales y ayuntamientos. Sin embargo, se preguntó por elecciones en lo particular y no por los procesos concurrentes en su conjunto. Como se advertirá, en algunos casos podrán hacerse comparaciones de un mismo tipo de elección entre estados e inter temporales, mientras que en otros, la intermedia especialmente, sólo podrá compararse entre estados.

Ahora presentamos los resultados más recientes en torno a la calidad de las elecciones para gobernador y de jefe de gobierno en 31 estados de la República y el Distrito Federal, respectivamente. En esta ocasión empezamos describiendo una batería de seis preguntas a propósito de posibles irregularidades y terminamos concentrándonos en dos, una sobre compra y otra sobre coacción de votantes. Las irregularidades sobre las que se preguntó a los expertos fueron, acerca de la votación de los muertos; las prácticas de doble voto o carrusel, como se le conoce en México al voto múltiple de partidarios o individuos contratados por el partido y/o candidato para tal efecto; también sobre la anulación de los votos recibidos por el (los) partido(s), cuando no había presencia de sus respectivos representantes en las casillas, el proceder de los partidarios de un candidato y/o partido, con posiciones de gobierno o no, en cuanto a recoger credenciales de elector de posibles votantes de sus rivales, prácticas de compra de votos y coacción a electores (véase anexo único).

El valor de las medias nacionales para cada una de estas prácticas fraudulentas, parece jerarquizar válidamente las problemáticas que enfrentan los sistemas electorales locales (¿acaso también el federal?), ${ }^{10}$

${ }^{10}$ El número de expertos entrevistados en cada estado aparece en el cuadro anexo. Aunque hay variación en la $n$ por entidad, al momento de estimar los promedios nacionales tomamos como unidad de observación al estado, es decir, se trata de un promedio de entidades de la República y no de los 222 individuos entrevistados. 
Esta revista forma parte del acervo de la Biblioteca Jurídica Virtual del Instituto de Investigaciones Jurídicas de la UNAM

así como su extensión y/o gravedad. Para evaluar la presencia de cada práctica, los entrevistados tuvieron cuatro opciones de respuesta: si consideraban que la irregularidad no se practicaba o era irrelevante, el valor de la respuesta fue uno; en el segundo caso, cuando era muy frecuente, extendida o relevante, el valor fue cinco. Entonces mientras más se acerque el promedio a uno, menos presente es la irregularidad que se explora.

A nivel nacional, la irregularidad que los expertos juzgaron menos frecuente fue que los muertos voten; le siguió en importancia el voto doble o múltiple, es decir, el carrusel. En tercer lugar figuró la anulación de votos en casillas del partido o candidato sin representante. Estas tres prácticas tuvieron un resultado por debajo del valor intermedio, por lo que no parecen graves aunque haya algunas excepciones estatales que ahora no comentaremos pero que pueden buscarse en el cuadro anexo. Con medias por arriba del valor intermedio, la cuarta irregularidad en importancia por su frecuencia según el juicio de nuestros expertos, fue recoger credenciales de elector a partidarios del candidato rival, le siguió en quinto sitio coaccionar votantes y con un valor promedio de cuatro, es decir, muy cerca del máximo posible, la compra de votos, en otras palabras, si los muertos no entran al juego, los vivos sí comercian con su voto (cuadro anexo 1).

Al sumar los valores de cada una de estas prácticas y dividir el resultado entre seis (para preservar la escala 1-5), obtuvimos un índice muy sencillo de presencia de irregularidades en las elecciones para gobernador en México 2001-2012. Lo que la medida expresa es la frecuencia de las irregularidades en su conjunto en cada estado y en un promedio nacional, según los expertos en política estatal.

Conforme a los valores del índice, Yucatán es el estado que tuvo más irregularidades en su última elección para gobernador, seguido de Chiapas, Chihuahua, Veracruz y Tabasco, todos ellos con valores por arriba de 3.5. Por debajo de esta cifra pero arriba del valor intermedio de tres, aparecen Oaxaca, Campeche, Puebla, Sonora, Jalisco, Nayarit, Durango y Zacatecas. Si asumimos que por arriba del valor intermedio, las irregularidades fueron muchas, estaríamos ante los trece estados en que los comicios pasados fueron de más baja calidad democrática, pese a lo cual, varios de los candidatos de las oposiciones locales, en alianzas multipartidistas o no, alcanzaron la gubernatura (gráfica 1). 
Esta revista forma parte del acervo de la Biblioteca Jurídica Virtual del Instituto de Investigaciones Jurídicas de la UNAM

Por el contrario, el Distrito Federal fue la entidad que a juicio de los expertos tuvo menos irregularidades en sus pasadas elecciones de jefe de gobierno; le siguieron Colima, Guerrero, Morelos, Nuevo León, Querétaro, Baja California, Sinaloa, San Luis Potosí, Quintana Roo, Guanajuato, Michoacán y el Estado de México. Todavía con valores por debajo del intermedio de tres pero muy cerca o ligeramente arriba de la media nacional de 2.8, figuraron Baja California Sur, Hidalgo, Tlaxcala y Tamaulipas (gráfica 1).

Por supuesto, el índice es apenas una primera aproximación al proceso electoral. El tratamiento cuidadoso y exhaustivo de la información en el cuestionario nos proporcionará una imagen completa de la calidad de los procesos electorales en cada estado. Y en el análisis y las explicaciones, podríamos responder en qué tipo de irregularidades suelen incurrir los diferentes partidos, los gobiernos y las oposiciones, cuál es el papel de las autoridades electorales y gubernamentales, así como del sistema político en su conjunto.

Gráfica 1. Irregularidades en elecciones de gobernador México, $2001-2012$

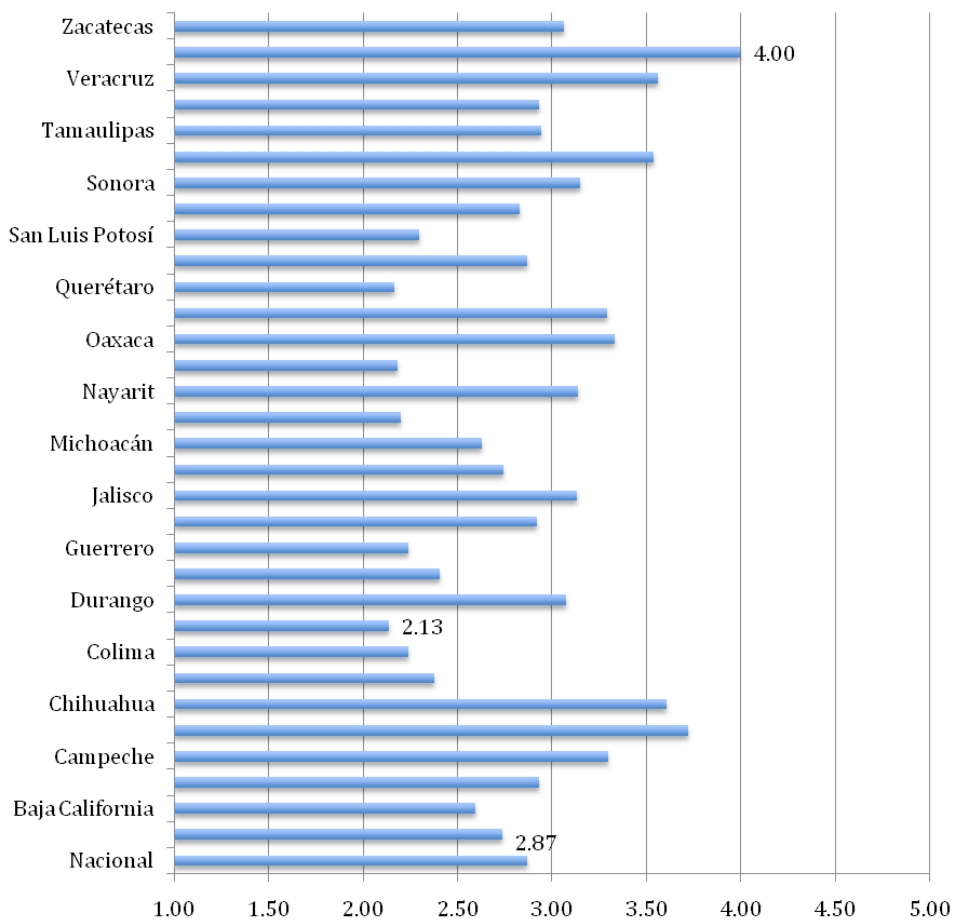


Esta revista forma parte del acervo de la Biblioteca Jurídica Virtual del Instituto de Investigaciones Jurídicas de la UNAM

Como apuntamos anteriormente, los expertos en política subnacional coinciden en que las irregularidades más recurrentes en las pasadas elecciones de gobernador, comprendidas en el periodo que abarca de 2001 a 2012, fueron, en primer lugar, la compra de votos y en segundo término, la coacción de electores. El diagnóstico converge con el tema de la poselección federal de 2012 en que tanto el Partido de la Revolución Democrática (PRD), como el Partido Acción Nacional (PAN) y sus candidatos a la Presidencia de la República, acusaron al ganador, es decir, al Partido Revolucionario Institucional (PRI) y su candidato, de haber incurrido de forma muy extendida en esas prácticas.

En su sentido más amplio, entendemos por clientelismo un método de movilización política que consiste en intercambiar bienes por votos, en una relación asimétrica en que los políticos, a los que usualmente se les dice patrones, utilizan la regla "si lo quieres, apóyame" (un condicional, "si y sólo si") como criterio de distribución de bienes para los electores, convertidos en consecuencia en clientes. ${ }^{11} \mathrm{El}$ intercambio clientelar, tiene un componente de coacción, es decir, de "fuerza o violencia" para obtener una conducta, aunque hay otro elemento, el voluntario que lo hace comparable a una transacción de mercado. Condicionar un bien gubernamental al sentido del voto, es una forma de coacción que sin embargo tiene variaciones en su intensidad y formas: se expresa claramente en su violencia, cuando el bien que se intercambia por el voto es la permanencia o movilidad de un individuo en un trabajo, la continuación en la provisión de un servicio colectivo como el suministro de agua potable a una comunidad o el acceso a los servicios de salud de un grupo o una persona; parece una transacción libre, cuando el bien intercambiado por el voto es una recompensa material de una sola vez que puede ir de un sencillo "promocional" a un kit de autoconstrucción residencial, o cuando se argumenta que la continuación en la provisión derivará de las diferencias en la jerarquización y diseño de políticas.

Tipificar primero compra y coacción de voto, y reconocerlo después en el curso de la vida política no es sencillo ni se trata de irregularidades siempre autoevidentes. En una transacción de compra, el votante y el patrón pueden argumentar que previamente existía afinidad partidaria y que sólo se reconoce la lealtad del partisano. La secrecía

11 Stokes, Susan, "Political Clientelism", en Boix, Carles y C. Stokes, Susan (eds.), Oxford University Press Handbook of Comparative Politics, 2006, pp. 604-627. 
Esta revista forma parte del acervo de la Biblioteca Jurídica Virtual del Instituto de Investigaciones Jurídicas de la UNAM

del voto también brinda al elector una salida anónima en comunidades relativamente grandes de votantes y ciertamente es un dispositivo contra la compra y la coacción, por lo que, si no se demuestra la violación de este precepto, será muy difícil probar el vínculo clientelar.

En la legislación mexicana, compra y coacción son delitos electorales penales tipificados en el título 24 del Código Penal Federal, en el que también se incluyen delitos en materia del Registro Nacional de Ciudadanos. Este título va de los artículos 401 al 413. El artículo 403 señala doce ilícitos en que pueden incurrir los ciudadanos, el 404 refiere a ministros de culto religioso, el 405 a funcionarios electorales, el 406 y 412 a funcionarios partidistas, el 407 a servidores públicos, el 408 a legisladores electos que sin razón no se presenten en sus cámaras, y los artículos 408 al 411 se refieren al Registro Nacional de Ciudadanos o al Registro Federal Electoral.

Con base en los tipos penales de este título, podrían considerarse conductas ilegales asociadas a clientelismo, patronazgo, compra y coacción de voto en su sentido más amplio, las previstas en los artículos 403 incisos III (presionar en la casilla o en la fila a electores para que voten por determinada opción), VI (solicitar votos por paga, dádiva, promesa de dinero u otra recompensa), VII (violar la secrecía del voto), IX (transportar votantes coartando su libertad de elección) y XI (solicitar u obtener declaración firmada del sentido del voto, o compromiso de éste, mediante amenaza o promesa de paga o dádiva); 404, pues la posible inducción del voto por parte de ministros del culto implica el intercambio de un bien privado (la salvación u otro bien espiritual que se obtendrá si y sólo si...) al votar por determinado partido o candidato; 405 inciso VI, que prevé la conducta del servidor público que en uso de sus funciones, induzca el voto de los electores al interior de la casilla o en la fila); 406 inciso I que describe la conducta de inducción de voto en casilla o en la fila por parte de funcionarios partidistas o candidatos; 407, que tipifica conductas de servidores públicos en sus incisos I (obligar a subordinados, haciendo uso de su jerarquía o autoridad, a votar de determinada manera) y II (condicionar la prestación de un servicio, el cumplimiento de programas o la realización de una obra pública, a que se vote de determinada manera).

¿Cuál ha sido la incidencia de estas dos irregularidades, compra y coacción, en elecciones estatales en el México del siglo XXI? El promedio nacional de la incidencia de compra de voto fue de 4 y para coacción de electores de 3.7, ambos por arriba del valor intermedio de la escala 
Esta revista forma parte del acervo de la Biblioteca Jurídica Virtual del Instituto de Investigaciones Jurídicas de la UNAM www.juridicas.unam.mx

http://biblio.juridicas.unam.mx

DOI: http://dx.doi.org/10.22201/iij.24487910e.2013.4.10043

y lejos de las prácticas irregulares menos extendidas. Chiapas fue la entidad en que ambas prácticas alcanzaron, conforme al juicio de los expertos, mayor relevancia, en el tope de su valor posible. Atrás de Chiapas, pero con muy extendidas prácticas de compra y coacción a juicio de los expertos (valores por arriba de 4.5 para ambas irregularidades), figuraron Veracruz, Chihuahua y Yucatán. En donde la compra de voto fue muy extendida pero no así la coacción, aparecen Zacatecas, Sonora, Quintana Roo, Nayarit, Nuevo León, y Jalisco. Por el contrario, sólo en Guanajuato la coacción de electores superó la compra de votos (gráfica 2).

\section{Gráfica 2. Compra y coacción de voto en elecciones de gobernador. México, 2000-2010}

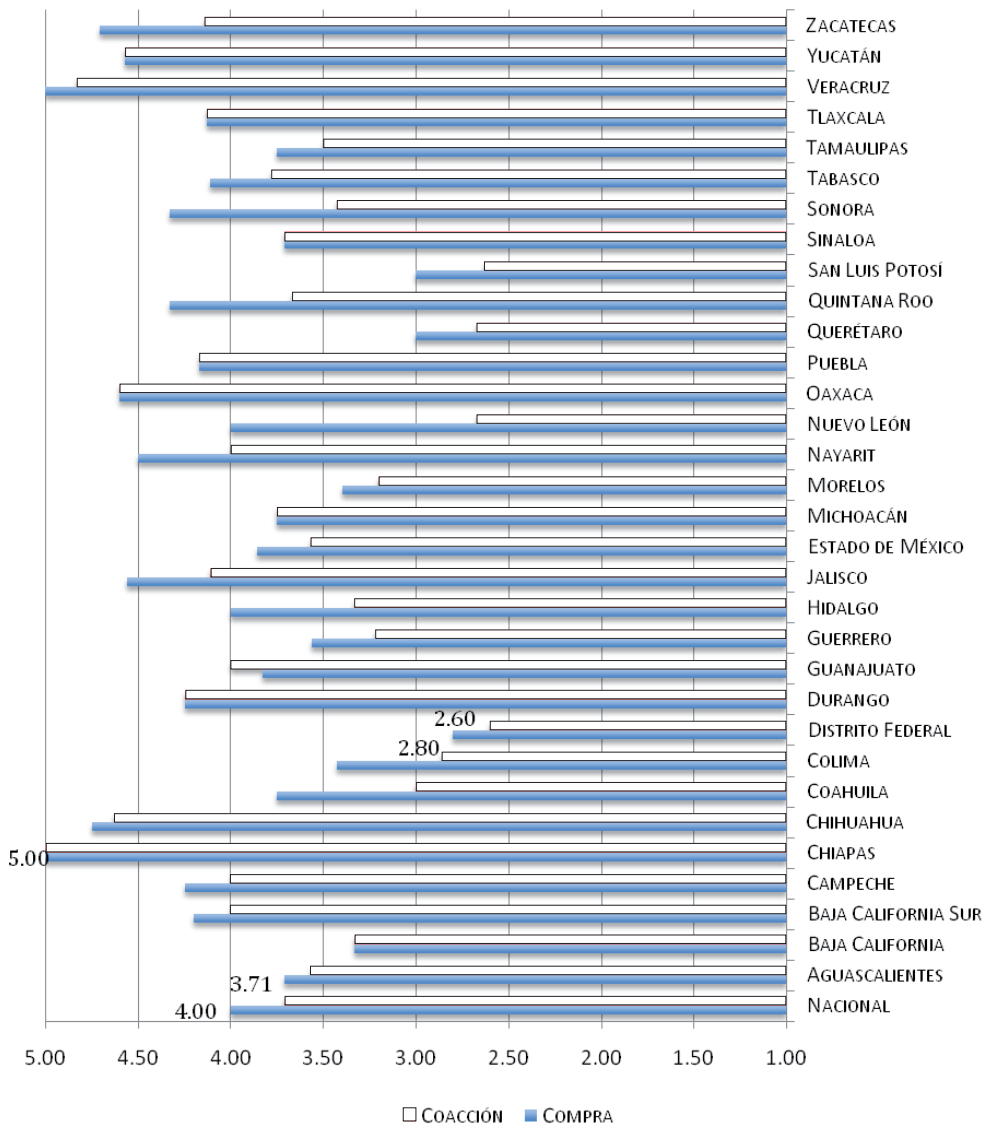


En la acera contraria, es decir, de los estados en que a juicio de los expertos éstas prácticas irregulares están menos extendidas, se encuentra en primer lugar, para ambas, el Distrito Federal, con un valor de 2.6 para coacción y 2.8 para compra, le siguen entidades como Querétaro y San Luis Potosí, con valores de 3 o menos para ambas prácticas, nótese, además, que su frecuencia en las entidades en que son menos difundidas, rebasa las medias nacionales de las otras irregularidades exploradas y que devienen menos relevantes, es decir, las de anular votos de partidos sin representante en la casilla, recoger credenciales a electores que se presume contrarios, y hacer que los muertos voten. Dicho de otra forma, aunque en el Distrito Federal, Querétaro y San Luis Potosí haya menos compra y coacción de voto que en las demás entidades del país, se trata de una irregularidad muy extendida y mucho más frecuente que otras posibles; es más, 29 estados del país alcanzan valores, por arriba de su valor intermedio.

A juicio de los expertos en política estatal, las elecciones de gobernador en México durante la primera década del nuevo siglo presenciaron extendidas prácticas clientelares, y en la elección presidencial de 2012 el problema de la compra y coacción de voto se convirtió en uno de los ejes del debate poselectoral. Indudablemente, la difusión de estos vicios de la democracia estimulará la presentación de iniciativas para acotarlos, tal vez por la vía de reformas legales que incrementen los castigos y la oportunidad para aplicarlos, mejoren los tipos penales, actúen sobre las fuentes de recursos, o las tres cosas a la vez. La información reunida en la investigación de que ahora hemos dado cuenta, nos permitirá conocer con mayor detalle las pautas de ocurrencia de estas irregularidades, si se presentan de forma diferente cuando un mismo partido está en el ejecutivo en un estado y/o la federación, y en la oposición en otro estado, si existen diferencias entre los estados gobernados por una u otra fuerza política, si la generalización de estas prácticas tiende a volverlas inocuas en el resultado final, o si hay un ganador sistemático de este tipo de prácticas.

\section{Fuentes consultadas}

Código Penal Federal, México, http://info4.juridicas.unam.mx/ijure/ $\mathrm{fed} / 8 /$. 
Esta revista forma parte del acervo de la Biblioteca Jurídica Virtual del Instituto de Investigaciones Jurídicas de la UNAM

Gervasoni, Carlos, "Democracia, autoritarismo e hibridez en las provincias argentinas: la medición y causas de los regímenes subnacionales", Journal of Democracy en Español, vol. 3, 2011.

Martí I Puig, Salvador et al. (eds.), La democracia en México. Un análisis a 10 años de alternancia, Barcelona, Bellaterra, 2011.

McMann, Kelly, Economic Autonomy and Democracy. Hybrid Regimes in Russia and Kyrgyzstan, Nueva York, Cambridge University Press, 2006.

- - - y Nikolai, Petrov, "A Survey of Democracy in Russia's Regions", Post-Soviet Geography and Economics, 41(3), 2000.

Méndez, Irma y LozA, Nicolás (coords.), La calidad de las elecciones sub nacionales en México, México, Flacso-PNUD, 2013 (en prensa). Stokes, Susan, "Political Clientelism", en Borx, Carles y C. STокES, Susan (eds.), Oxford University Press Handbook of Comparative Politics, 2006. 


\section{Anexo único: irregularidades en las elecciones de gobernador evaluadas}

\begin{tabular}{|c|c|c|c|c|c|c|c|c|c|c|c|c|c|c|c|}
\hline & \multicolumn{2}{|c|}{$\begin{array}{l}\text { Que voten los } \\
\text { muertos }\end{array}$} & \multicolumn{2}{|c|}{$\begin{array}{c}\text { Doble voto o } \\
\text { carrusel }\end{array}$} & \multicolumn{2}{|c|}{$\begin{array}{l}\text { Anular votos } \\
\text { sin defensor }\end{array}$} & \multicolumn{2}{|c|}{$\begin{array}{c}\text { Recoger } \\
\text { credenciales }\end{array}$} & \multicolumn{2}{|c|}{$\begin{array}{l}\text { Comprar } \\
\text { votantes }\end{array}$} & \multicolumn{2}{|c|}{$\begin{array}{c}\text { Coaccionar } \\
\text { votantes }\end{array}$} & \multicolumn{2}{|l|}{1} \\
\hline & $n$ & Media & $D T$ & Media & $D T$ & Media & $D T$ & Media & $D T$ & Media & DT & Media & $D T$ & Media & $D T$ \\
\hline Nacional & 222 & 1.59 & 0.97 & 2.29 & 1.23 & 2.37 & 1.22 & 3.25 & 1.30 & 4.00 & 1.13 & 3.71 & 1.27 & 2.87 & 1.19 \\
\hline Aguascalientes & 7 & 1.00 & 0.00 & 2.00 & .83 & 3.00 & 1.55 & 3.14 & 1.08 & 3.71 & 1.13 & 3.57 & 1.42 & 2.74 & 1.00 \\
\hline Baja California & 6 & 1.40 & 0.89 & 2.00 & .89 & 2.50 & 1.52 & 3.00 & 1.26 & 3.33 & 1.21 & 3.33 & 1.21 & 2.59 & 1.16 \\
\hline $\begin{array}{ll}\text { Baja } & \text { California } \\
\text { Sur } & \\
\end{array}$ & 5 & 2.00 & 1.12 & 2.40 & .88 & 2.00 & 1.39 & 3.00 & 1.55 & 4.20 & .44 & 4.00 & 1.20 & 2.93 & 1.10 \\
\hline Campeche & 8 & 1.86 & 1.51 & 2.86 & 1.62 & 3.43 & 1.67 & 3.38 & 1.73 & 4.25 & 1.42 & 4.00 & 1.64 & 3.30 & 1.60 \\
\hline Chiapas & 7 & 2.00 & 1.58 & 3.17 & 1.75 & 3.33 & 1.23 & 3.83 & 1.50 & 5.00 & .00 & 5.00 & .00 & 3.72 & 1.01 \\
\hline Chihuahua & 8 & 2.88 & 1.02 & 3.63 & .53 & 2.38 & .53 & 3.38 & .76 & 4.75 & .47 & 4.63 & .53 & 3.61 & 0.64 \\
\hline Coahuila & 8 & 1.25 & 0.72 & 2.25 & 1.52 & 1.75 & 1.19 & 2.25 & 1.19 & 3.75 & 1.52 & 3.00 & 1.64 & 2.38 & 1.30 \\
\hline Colima & 7 & 1.00 & 0.00 & 1.43 & .80 & 1.71 & .77 & 3.00 & 1.17 & 3.43 & 1.29 & 2.86 & 1.08 & 2.24 & 0.85 \\
\hline Distrito Federal & 5 & 1.20 & 0.44 & 2.00 & .69 & 2.00 & .69 & 2.20 & .44 & 2.80 & .82 & 2.60 & .88 & 2.13 & 0.66 \\
\hline Durango & 6 & 1.20 & 0.45 & 2.50 & 1.00 & 2.25 & .96 & 4.00 & .82 & 4.25 & .96 & 4.25 & .96 & 3.08 & 0.86 \\
\hline Guanajuato & 7 & 1.14 & 0.38 & 1.14 & .38 & 1.14 & .38 & 3.17 & 1.63 & 3.83 & 1.63 & 4.00 & 1.29 & 2.40 & 0.95 \\
\hline Guerrero & 9 & 1.00 & 0.00 & 1.33 & .73 & 2.11 & 1.09 & 2.22 & 1.13 & 3.56 & 1.17 & 3.22 & 1.24 & 2.24 & 0.89 \\
\hline Hidalgo & 6 & 1.67 & 1.21 & 2.33 & .82 & 2.50 & 1.05 & 3.67 & 1.03 & 4.00 & .89 & 3.33 & 1.03 & 2.92 & 1.01 \\
\hline Jalisco & 9 & 2.00 & 0.67 & 2.29 & .99 & 2.57 & 1.02 & 3.25 & 1.33 & 4.56 & .75 & 4.11 & 1.31 & 3.13 & 1.01 \\
\hline $\begin{array}{l}\text { Estado } \\
\text { México }\end{array}$ & 7 & 1.29 & 0.77 & 2.14 & .91 & 2.29 & .77 & 3.29 & 1.13 & 3 & 1.08 & 3.57 & 1.29 & 2.74 & 0.99 \\
\hline Michoacán & 8 & 1.75 & 0.72 & 1.75 & .72 & 1.88 & 1.15 & 2.88 & 1.49 & 3.75 & .91 & 3.75 & .72 & 2.63 & 0.95 \\
\hline Morelos & 5 & 1.00 & 0.00 & 1.20 & .44 & 1.20 & .44 & 3.20 & 1.28 & 3.40 & 1.12 & 3.20 & 1.28 & 2.20 & 0.76 \\
\hline Nayarit & 6 & 1.50 & 0.84 & 2.83 & 1.47 & 2.50 & 1.64 & 3.50 & 1.38 & 4.50 & .55 & 4.00 & 1.10 & 3.14 & 1.16 \\
\hline
\end{tabular}




\begin{tabular}{|l|c|c|c|c|c|c|c|c|c|c|c|c|c|c|c|c|}
\hline \multicolumn{2}{|c|}{} & \multicolumn{2}{c|}{$\begin{array}{c}\text { Que voten los } \\
\text { muertos }\end{array}$} & \multicolumn{2}{c|}{$\begin{array}{c}\text { Doble voto o } \\
\text { carrusel }\end{array}$} & \multicolumn{2}{|c|}{$\begin{array}{c}\text { Anular votos } \\
\text { sin defensor }\end{array}$} & \multicolumn{2}{c|}{$\begin{array}{c}\text { Recoger } \\
\text { credenciales }\end{array}$} & \multicolumn{2}{c|}{$\begin{array}{c}\text { Comprar } \\
\text { votantes }\end{array}$} & \multicolumn{2}{c|}{$\begin{array}{c}\text { Coaccionar } \\
\text { votantes }\end{array}$} & \multicolumn{2}{|c|}{$I$} \\
\hline & $n$ & Media & $D T$ & Media & DT & Media & DT & Media & DT & Media & DT & Media & DT & Media & DT \\
\hline Nuevo León & 7 & 1.00 & 0.00 & 1.14 & .38 & 1.71 & .77 & 2.57 & 1.29 & 4.00 & 1.17 & 2.67 & 1.39 & 2.18 & 0.83 \\
\hline Oaxaca & 5 & 2.00 & 1.20 & 2.00 & 1.20 & 2.60 & .88 & 4.20 & .82 & 4.60 & .54 & 4.60 & .54 & 3.33 & 0.86 \\
\hline Puebla & 6 & 2.17 & 0.98 & 2.20 & .84 & 3.20 & 1.10 & 3.83 & 1.17 & 4.17 & 1.17 & 4.17 & .98 & 3.29 & 1.04 \\
\hline Querétaro & 6 & 1.33 & 0.52 & 1.83 & 1.17 & 1.83 & .75 & 2.33 & .52 & 3.00 & .63 & 2.67 & .52 & 2.17 & 0.69 \\
\hline Quintana Roo & 6 & 1.20 & 0.45 & 1.80 & .84 & 2.20 & .84 & 4.00 & .63 & 4.33 & 1.21 & 3.67 & 1.75 & 2.87 & 0.95 \\
\hline San Luis Potosín & 8 & 1.13 & 0.36 & 2.13 & 1.39 & 2.25 & 1.80 & 2.63 & 1.64 & 3.00 & 1.73 & 2.63 & 1.54 & 2.30 & 1.41 \\
\hline Sinaloa & 7 & 1.71 & 1.13 & 2.57 & 1.42 & 2.14 & .91 & 3.14 & 1.60 & 3.71 & 1.13 & 3.71 & 1.27 & 2.83 & 1.24 \\
\hline Sonora & 7 & 1.33 & 0.83 & 3.29 & 1.52 & 3.17 & 1.50 & 3.33 & 1.89 & 4.33 & 1.66 & 3.43 & 1.42 & 3.15 & 1.47 \\
\hline Tabasco & 9 & 3.00 & 1.63 & 3.78 & 1.13 & 2.78 & 1.34 & 3.78 & 1.13 & 4.11 & 1.09 & 3.78 & 1.00 & 3.54 & 1.22 \\
\hline Tamaulipas & 8 & 2.00 & 1.55 & 2.88 & 1.85 & 2.63 & 1.64 & 2.88 & 1.59 & 3.75 & 1.62 & 3.50 & 1.90 & 2.94 & 1.69 \\
\hline Tlaxcala & 8 & 1.57 & 0.81 & 2.14 & .93 & 2.13 & .86 & 3.50 & 1.55 & 4.13 & 1.02 & 4.13 & 1.02 & 2.93 & 1.03 \\
\hline Veracruz & 7 & 1.20 & 0.46 & 3.17 & 1.50 & 3.17 & 1.19 & 4.00 & 1.11 & 5.00 & .00 & 4.83 & .42 & 3.56 & 0.78 \\
\hline Yucatán & 7 & 2.80 & 1.12 & 3.60 & .56 & 4.00 & 1.29 & 4.43 & .80 & 4.57 & .80 & 4.57 & .80 & 4.00 & 0.90 \\
\hline Zacatecas & 7 & 1.57 & 0.54 & 2.14 & 1.36 & 2.25 & .99 & 3.57 & 1.53 & 4.71 & .49 & 4.14 & 1.48 & 3.06 & 1.07 \\
\hline
\end{tabular}

Pregunta 31: A veces los partidos recurren a prácticas tales como "que voten los muertos", que sus militantes voten dos veces con credenciales falsas o hagan "carrusel", anular votos de los partidos que no tienen representante en la casilla, recoger con engaños la credencial de elector de potenciales votantes del adversario, comprar o coaccionar a los votantes u otras. ¿Diría que en las elecciones estatales de [año] hubo ninguna, pocas, algunas, bastantes o muchas irregularidades de este tipo? (1 fue que no hubo y 5 que hubo muchas). 